\title{
Low false alarm attackers detection in RPL by considering timing inconstancy between the rank measurements
}

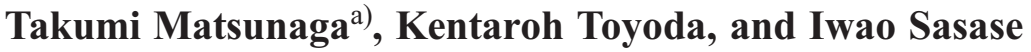 \\ Dept. of Information and Computer Sciences, Keio University, \\ 3-14-1 Hiyoshi, Kohoku, Yokohama 223-8522, Japan \\ a)matsunaga@sasase.ics.keio.ac.jp
}

Abstract: We propose a low false alarm attackers detection in the IPv6 Routing Protocol for Low-Power and Lossy Networks (RPL) by considering timing inconstancy between rank measurements. In the proposed scheme, each node sends the latest rank broadcasted to neighbor nodes instead of its current rank to a sink so as to avoid the rank mismatch due to timing inconstancy. We also introduce the timestamp for reporting rank measurements to decrease the false alarm due to packet loss. Simulation results show that the proposed scheme reduces the false alarm rate.

Keywords: IoT, RPL, security, sensor network

Classification: Network

\section{References}

[1] K. Ashton, "That 'Internet of Things' thing," RFID Journal, http://www. rfidjournal.com/articles/view?4986, 2009.

[2] T. Winter, P. Thubert, A. Brandt, J. Hui, R. Kelsey, P. Levis, K. Pister, R. Struik, J. Vasseur, and R. Alexander, "RPL: IPv6 routing protocol for low-power and lossy networks," RFC 6550, http://tools.ietf.org/html/rfc6550, 2012.

[3] A. Le, J. Loo, A. Lasebae, A. Vinel, Y. Chen, and M. Chai, "The impact of rank attack on network topology of routing protocol for low-power and lossy networks," IEEE Sensors J., vol. 13, no. 10, pp. 3685-3692, 2013. DOI:10. 1109/JSEN.2013.2266399

[4] S. Raza, L. Wallgren, and T. Voigt, "SVELTE: Real-time intrusion detection in the Internet of Things," Ad Hoc Netw., vol. 11, no. 8, pp. 2661-2674, 2013. DOI:10.1016/j.adhoc.2013.04.014

[5] C. Karlof and D. Wagner, "Secure routing in wireless sensor networks: attacks and countermeasures," Ad Hoc Netw., vol. 1, no. 2, pp. 293-315, 2003. DOI:10. 1016/S1570-8705(03)00008-8

[6] F. Osterlind, A. Dunkels, J. Eriksson, N. Finne, and T. Voigt, "Cross-level sensor network simulation with COOJA," IEEE Conference on Local Computer Networks (LCN), pp. 641-648, 2006. DOI:10.1109/LCN.2006.322172

[7] O. Gnawali and P. Levis, "The minimum rank with hysteresis objective function," RFC 6719, http://tools.ietf.org/html/rfc6719, 2012. 


\section{Introduction}

In the Internet of Things (IoT), resource-constrained sensing devices are connected to the Internet via IPv6 network so as to monitor and control everything e.g., energy consumption [1]. The IPv6 Routing Protocol for Low-Power and Lossy Networks (RPL) is a standard routing protocol to realize the IoT [2]. In the RPL, nodes create a loop-free tree-based topology which is called as Destination-Oriented Directed Acyclic Graph (DODAG) to communicate each other. In order to construct the tree topology, each node has a rank. The rank is cumulative value e.g., the numbers of hops from the sink, and the sink and nodes periodically broadcast a DIO (DODAG Information Object) message to inform their ranks. Each node selects the least rank neighbor node as its parent node.

However, a (malicious) node can claim a forged rank that is lower than the real one to collect more packets from child nodes [3]. Raza et al. proposed a real-time intrusion detection scheme, which is referred to SVELTE, to detect such malicious nodes [4]. In SVELTE, the sink collects rank information from each node e.g., their neighbor and parent ranks, and then detects any rank inconsistency which implies a potential forged rank attacker. However, SVELTE has a problem that false alarm rate is high. This is because it does not consider timing consistency or packet loss.

In this paper, we propose a low false alarm attackers detection in the RPL by considering timing inconsistency between the rank measurements. The first proposal is that the sink lets each node send the latest rank broadcasted to neighbor nodes instead of its current rank so as to collect ranks at the same time. The second proposal is that the sink lets each node attach a timestamp when each node sends and receives ranks in order for the sink to change the threshold with respect to the timing inconsistency.

We evaluate the false alarm rates by computer simulation and show that the proposed scheme reduces the false alarm rate in comparison with SVELTE.

\section{Attacker model}

We assume that an attacking node can claim fake rank that is lower rank than real rank to collect more packets from child nodes. More specifically, we assume that the attacking node claims rank 0 which is the same as that of the sink (= minimum rank). By claiming as the lowest rank, the attacking node can launch the selective forward attack and the packet drop attack [5].

\section{Conventional scheme}

SVELTE detects attacking nodes by finding rank inconsistency in the network. In the RPL, the parent rank must be lower than its child nodes: thus the sink judges the node as an attacker if its rank is lower than its parent node. Therefore, the sink requests every node to report its own rank and neighbor nodes' ranks. Receiving a request, each node responds with its neighbor and parent ranks.

At the second module, the sink analyzes the collected data and detects attacking nodes. The sink checks each node's rank inconsistency by comparing the rank that it insists with the rank that its neighbors report. The sink judges that a node is an attacker if the difference between the ranks is larger than the pre-defined threshold. 


\subsection{Problems of SVELTE}

SVELTE has a problem that false alarm rate is high. There are two situations that false alarm occurs.

Problem 1: The first situation is when a sink sends a request just before a node broadcasts a DIO message. In this case, the node sends the rank at time $t$, whereas its neighbor node sends the previous rank at $t-1$ since neighbors have not received its rank at $t$.

Problem 2: The second one is when neighbor node fails to receive a DIO message due to packet loss. In this case, the node sends the rank at time $t$, however, its neighbor node sends the rank at $t-1$ since it cannot receive the updated DIO message.

These two problems bring about high false alarm rate that the sink mistakenly judges nodes as attackers.

\section{Proposed scheme}

Here we propose a low false alarm attackers detection in the RPL by considering timing inconstancy between rank measurements. We improves SVELTE with two modifications.

\subsection{Proposal 1: SVELTE with same time rank}

In order to solve the Problem 1, the sink lets each node retain the latest rank broadcasted to neighbor nodes and then report this rank instead of its current rank so as to avoids the rank mismatch due to timing inconstancy.

We show an example of Proposal 1 in Fig. 1(a). We consider the case that the node 1 broadcasts a DIO message with its rank 1 's rank $=3$ and its neighbor nodes receive the rank 1's rank $=3$. Then, the node 1 updates its rank 1's current rank $=$ 4. In the proposed scheme, the node 1 reports 1 's rank $=3$ instead of 1 's current rank $=4$ to the sink so that the sink can compare it with the same time ranks reported by 1's neighbors. This way reduces unnecessary false alarm caused by timing inconsistency.

\subsection{Proposal 2: SVELTE with timestamp}

In order to mitigate the Problem 2, each node attaches a timestamp when it sends and receives DIO messages and also reports timestamps to the sink in order for the sink to take timing inconstancy into consideration due to packet loss. It does not need strict time synchronization since the minimum interval of DIO message is relatively long (about $4 \mathrm{sec}$ ) [2]. If these timestamps are the same, the sink uses threshold $k_{1}$, otherwise the sink use threshold $k_{2}\left(>k_{1}\right)$ instead.

We show an example of Proposal 1 in Fig. 1(b). We consider the case that the node 1 broadcasts its rank $1^{\prime} s$ current rank $=4$ in DIO message at $t_{6}$, though its neighbor nodes cannot receive this DIO message due to packet loss. In this case, the neighbor nodes retain the 1 's one time older $\left(t=t_{3}\right)$ rank 1 's rank $=3$. In the proposed scheme, the node 1 and the neighbor nodes attach timestamp $t_{6}$ and $t_{3}$ and send them. The sink sets $k_{2}$ as the threshold because the time difference between them is not $0\left(t_{6}-t_{3} \neq 0\right)$. Therefore, it is possible to reduce false alarm rate in order to take timestamps into consideration. 


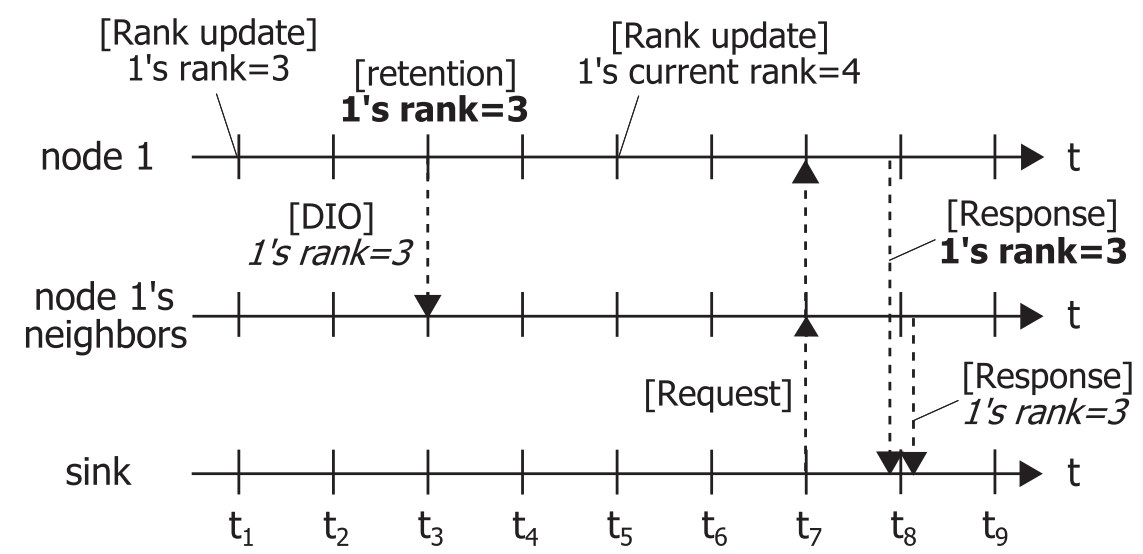

(a) Example of Proposal 1.

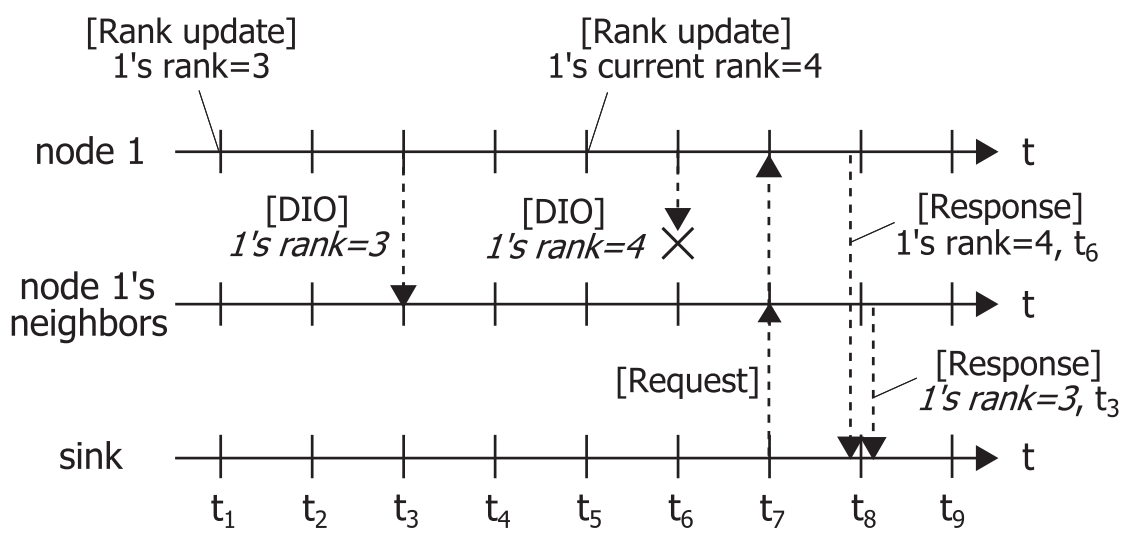

(b) Example of Proposal 2.

Fig. 1. Example that proposed scheme solves the timing inconsistency.

\section{Evaluation}

\subsection{Simulation model}

We evaluate the false alarm rate for SVELTE, our Proposal 1, Proposal 2, and Proposal 1\&2 in two packet loss rate (high and low) with Cooja [6]. The false alarm rate denotes the ratio of the total number of unsuccessful detections to the total number of detections. We show the simulation parameters in Table I. In this radio model, reception probability is decreased according to the square of the distance. These thresholds $k_{1}, k_{2}$ are based on empirical evaluation as well as the conventional scheme. The attackers can collect packets which all nodes with the exception of the sink's neighbor node send even if the number of attackers is limited. Therefore, we assume that the maximum number of attackers is four.

\subsection{Simulation results and discussion}

Fig. 2 shows false alarm rate versus the number of attackers. As shown in Fig. 2, the Proposal 1 and Proposal 2 achieve lower false alarm rate than SVELTE against any number of attackers and packet loss rate. The Proposal $1 \& 2$ achieves much lower false alarm rate than solely used Proposal 1 and Proposal 2 because they mitigate different roots of the problems in SVELTE.

The difference between the false alarm rate in high and low packet loss rate with the proposed scheme is lower than the difference with SVELTE. The 
Table I. Simulation parameters

\begin{tabular}{cc}
\hline Name & Value \\
\hline Simulator & Cooja \\
Simulation area & $400 \mathrm{~m} \times 400 \mathrm{~m}$ \\
Number of all nodes & 32 \\
Number of sink & 1 \\
Number of attackers & $1-4$ \\
Transmission range & $100 \mathrm{~m}$ \\
Interference range & $120 \mathrm{~m}$ \\
Radio model & Unit Disk Graph Medium: Distance Loss \\
Transfer success ratio & $100 \%$ (high and low) \\
Receiver success ratio & $80 \%$ (high), 50\% (low) \\
Objective function & Minimum Rank \\
Threshold & with Hysteresis Objective Function [7] \\
& $k_{1}=0.2, k_{2}=0.4$ \\
\hline
\end{tabular}

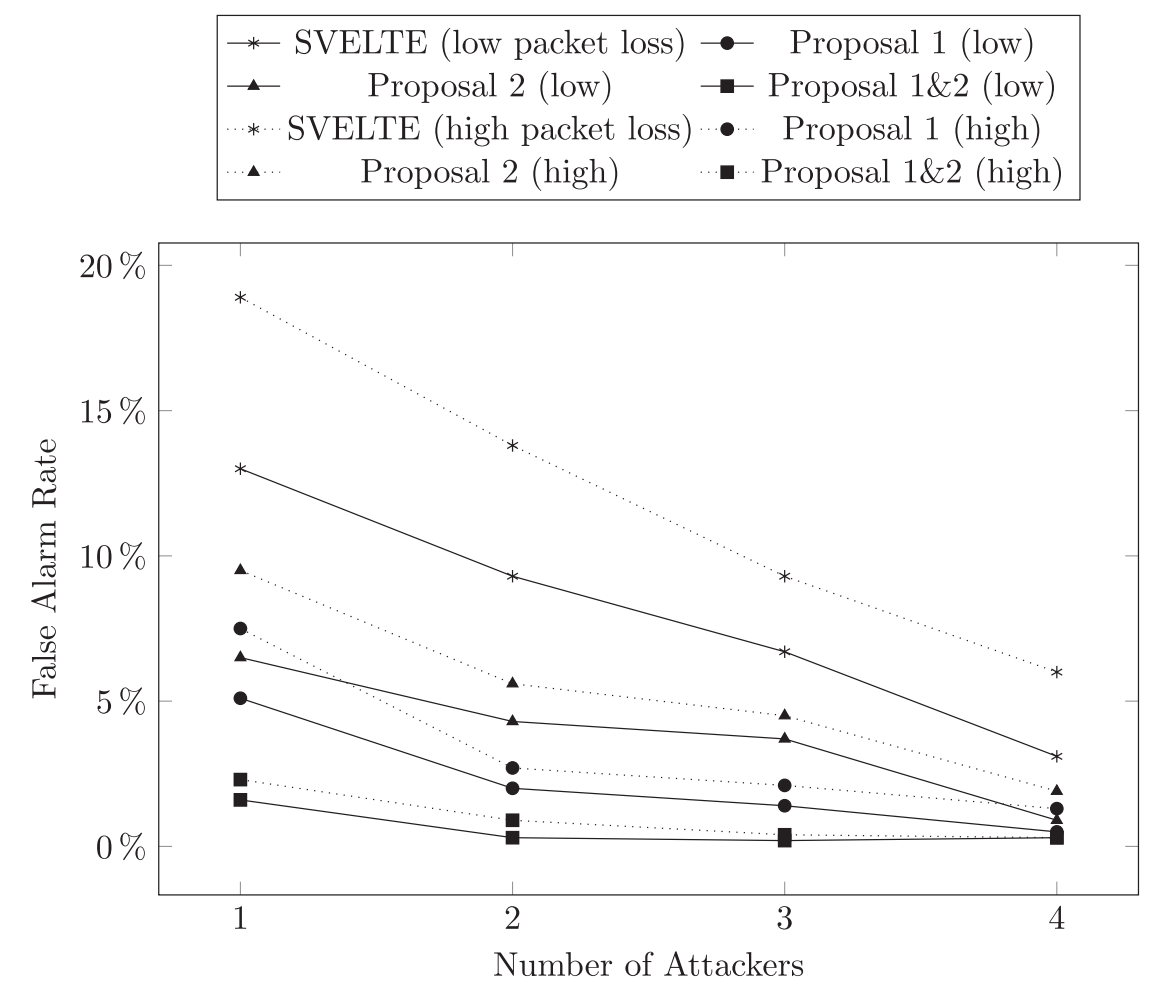

Fig. 2. False alarm rate versus the number of attackers in low and high packet loss rate.

Proposal 1 can reduce the false alarm since the higher the packet loss rate is, the larger time fluctuation of rank is [7].

When the number of attacker is 1 , the Proposal $1 \& 2$ can much reduce the false alarm rate by $11.4 \%$ (in low) or $16.6 \%$ (in high) in comparison with SVELTE. When the number of attacker is 4 , the Proposal $1 \& 2$ can reduce the false alarm rate by $2.8 \%$ or $5.7 \%$ in comparison with SVELTE. The false alarm rate decreases according to increased attackers so as to increase the nodes which have lower rank 
due to attackers' injurious claims (= rank 0). The node's rank nearby the sink and attackers tends to be stable compared to that in much far from the sink since they claim the constant rank 0 .

\section{Conclusion}

In this paper, we have proposed a low false alarm attackers detection in the RPL with two proposals. The first proposal is the sink collects the ranks at the same time between a node and its neighbor node. The second proposal is the sink takes timing inconstancy into consideration by introducing timestamps so as to reduce the false alarm. We show that the proposed scheme reduces the false alarm rate in comparison with SVELTE by computer simulation.

\section{Acknowledgments}

This work is partly supported by the Grant in Aid for Scientific Research (No. 26420369) from Ministry of Education, Sport, Science and Technology, Japan. 